\title{
Topological Hunds rules and the electronic properties of a triple lateral quantum dot molecule
}

\author{
Marek Korkusinski, ${ }^{1}$ Irene Puerto Gimenez, ${ }^{1}$ Pawel Hawrylak, ${ }^{1}$ Louis \\ Gaudreau, ${ }^{2,3}$ Sergei A. Studenikin, ${ }^{2}$ and Andrew S. Sachrajda ${ }^{2}$ \\ ${ }^{1}$ Quantum Theory Group, Institute for Microstructural Sciences, \\ National Research Council of Canada, \\ Ottawa, Ontario, Canada K1A OR6 \\ ${ }^{2}$ Quantum Physics Group, Institute for Microstructural Sciences, \\ National Research Council of Canada, \\ Ottawa, Ontario, Canada K1A OR6 \\ ${ }^{3}$ Régroupement Québécois sur les Matériaux de Pointe, \\ Université de Sherbrooke, Québec, Canada J1K 2R1
}

\begin{abstract}
We analyze theoretically and experimentally the electronic structure and charging diagram of three coupled lateral quantum dots filled with electrons. Using the Hubbard model and real-space exact diagonalization techniques we show that the electronic properties of this artificial molecule can be understood using a set of topological Hunds rules. These rules relate the multi-electron energy levels to spin and the inter-dot tunneling $t$, and control charging energies. We map out the charging diagram for up to $N=6$ electrons and predict a spin-polarized phase for two holes. The theoretical charging diagram is compared with the measured charging diagram of the gated triple-dot device.
\end{abstract}

PACS numbers: 73.21.La,73.23.Hk 


\section{INTRODUCTION}

Following on earlier work which showed that a small and well-controlled number of electrons can be confined in a single $\mathrm{e}^{1,2}$ and a double quantum dot, $\underline{3,4,5,6}, \underline{7}$ an artificial lateral quantum molecule consisting of three quantum-mechanically coupled lateral quantum dots has been demonstrated. $\frac{8}{-}$ The triple quantum dot molecule is a natural step toward creating quantum dot networks, with potential applications in quantum computing. $\underline{9}, 10,11$ When filled with three electrons, one electron per dot, this device can serve as a simple quantum logic circuit, with each electron spin treated as a qubit. One can also use the molecule as a single coded qubit, $, 11,12,13,14$ whose states are encoded in the states of three electronic spins but tunable with applied voltage. The triple dot could also be used to create entanglement between spin qubits, $\stackrel{15}{,}$ spin and charge qubits, $\frac{16}{2}$ as a charge rectifier, 17,18 and may exhibit a characteristic Kondo effect when coupled to the leads $\underline{19}, 20,21,22,23,24,25,26$ With electrons localized on individual dots and their tunneling controlled by gates, the triple dot molecule can be also thought of as an implementation of the tunable Hubbard model, an important step toward realization of "quantum materials", $\underline{27}, \underline{28}, \underline{29}, \underline{30}, 31, \underline{32}$

The electronic properties of the triple quantum dot with one electron per dot have been studied theoretically by a number of authors. To make contact with the pairwise-exchange formalism used in quantum information, 11 attempts were made to map the properties of this system onto those of the three-spin Heisenberg model. Scarola and Das Sarma ${ }^{33}$ used the Hubbard, variational, and exact diagonalization approaches to demonstrate that this mapping can be carried out only for a limited range of triple-dot parameters. Mizel and $\operatorname{Lidar}^{34,35,36}$ arrived at similar conclusions using the Heitler-London and Hund-Mülliken schemes to calculate the energy levels of three coupled dots with one electron per dot. In both cases the many-body effects were responsible for the appearance of higher-order terms in the effective spin Hamiltonian. In an alternative approach, in Ref. 12 we have used real-space wave functions and the configuration-interaction technique to analyze the three-electron triple-dot molecule acting as a single coded qubit and shown how its energy levels can be tuned by voltages applied to gates defining the structure.

Properties of the triple-dot molecule as a scattering center have also been studied using quantum transport techniques. Using the density-functional and quantum Monte Carlo methods, Stopa ${ }^{17}$ calculated the current flowing through a nominally empty molecule con- 
nected to electron reservoirs and under bias. The rectifying behavior of the system predicted in this analysis was confirmed experimentally. $\stackrel{18}{ }$ Landrón de Guevara and Orellana 37 calculated the zero-temperature conductance through a linear molecule coupled in parallel to the leads using a Hubbard approach in a magnetic field. Apart from the Fano resonances in the spectrum, they found evidence of formation of the quantum-molecular states decoupled from the leads. The Hubbard model has also been used to investigate the triple-dot system in the Kondo regime, both in the linear $\frac{20,21,22}{2}$ and triangular topology. 23,24,25,26

In this paper we describe the electronic properties of a lateral triple quantum dot molecule as a function of electron numbers. In analogy to the work on quantum materials, $\underset{27}{2} \underset{28}{2}$ we model our system with the Hubbard Hamiltonian, but the obtained results are verified by microscopic methods. In the Hubbard model we retain only one lowest-energy orbital per dot. The lowest-energy shell of the molecule can be filled with up to $N_{e}=6$ electrons. We analyze in detail the ordering of energy levels, the spacing of Coulomb blockade peaks and the charging and spin phase diagram of this shell. We demonstrate that the energy levels of the molecule are related to the total spin of electrons but not directly related to the charge e. We find the spin singlet as the two-electron ground state, with the singlet-triplet (S-T) splitting proportional to the single-particle tunneling matrix element $t$. This is in contrast to atoms, where the S-T splitting is proportional to the electronic exchange and hence to $e^{2}$, or to magnetic solids, where super-exchange leads to $\mathrm{S}-\mathrm{T}$ splitting proportional to $1 / e^{2}$. On the other hand, for two holes $\left(N_{e}=4\right)$ we predict a spin polarized ground state and a singlettriplet transition driven only by modifying the topology of the system. For three electrons in a half-filled shell $\left(N_{e}=3\right)$ we confirm the existence of the frustrated antiferromagnetic ground state. 12 The fact that the tunneling alone distinguishes singlet and triplet states is related to the interplay of the Fermi statistics and system topology. We term the set of rules established here and relating spin of the ground state to the filling of the shell, topology, and tunneling, "topological Hunds rules". The ability to tune tunneling by gates opens the possibility of directly manipulating the electron spin using electrical means only, of interest in designing novel quantum materials, magneto-electronics and quantum computation. We show that the Hubbard model is capable of reproducing the charging diagram of a lateral gated triple-dot measured recently by Gaudreau et al. $\underline{\underline{ }}$

The paper is organized as follows. In Sec. II we describe the model lateral triple-dot device and construct the Hubbard Hamiltonian. In Sec. III we determine the electronic 
structure of the device charged with $N_{e}=1$ to 6 electrons. Results of the Hubbard model are tested against real space (RSP) configuration interaction (RSP-CI) and linear combination of atomic or quantum dot orbitals (LCAO-CI) calculations. The charging diagram as a function of the dot energies is presented and analyzed in Sec. IV] In Sec. V] we relate the calculated and measured charging diagrams. Summary and conclusions are presented in Sec. VI.

\section{THE MODEL}

The proposed model gated triple-dot device realizing the triple dot using only metallic gates, studied in Ref. 12 and related to the one studied by Gaudreau et al. in Ref. 8, is shown in Fig. 1(a). It consists of a heterojunction with a two-dimensional electron gas (2DEG) created at a distance $D$ below the top surface of the sample. The metallic gates deposited on the surface serve to deplete the 2DEG underneath. Any opening in the gates is translated electrostatically into a local potential minimum, capable of confining a small number of electrons. Thus, in our model the three circular holes in the main gate (shown in gray) define a triangular triple quantum dot lateral confinement. Each isolated potential minimum gives rise to a quantized energy spectrum, of which we retain only the lowest energy level $E_{i}$ in dot $i$. By tuning the voltage on the main gate we can control the number of confined electrons. For example, in Fig. 1(a) we show $N_{e}=2$ electrons with parallel spins localized on two of the dots. This is not, however, a depiction of a quantum molecular state: due to the interdot coupling the electrons are delocalized across the molecule. The main gate alone defines a symmetric triangular molecule with identical pairwise coupling of all dots. This triple-dot potential can be well approximated by a sum of three Gaussians.

The single-particle confinement can be additionally tuned by three smaller gates, shown in red, green, and blue. Their arrangement with respect to the potential minima is shown schematically in Fig. 1(b). The gate $V G_{1}$ controls simultaneously the lowest energy levels $E_{1}$ and $E_{2}$ of dots 1 and 2 , and the gate $V G_{3}$ controls the energy level $E_{3}$ of dot 3 . Additionally, the gate $V G_{13}$ is designed to tune the topology of the system without significantly changing the energies $E_{i}$. By biasing it with a sufficiently high negative voltage we increase the tunneling barrier between dots 1 and 3 and change the sample layout from a closed triangle, in which all dots are identically coupled, to a linear molecule, in which the tunneling between 
dots 1 and 3 is not allowed.

We examine the electronic properties of our triple quantum dot molecule in the frame of the Hubbard model with one spin-degenerate orbital per dot. Without specifying them explicitly, the localized orbitals in the Hubbard model are assumed to be orthogonal. This is to be contrasted with the approach starting from the linear combination of atomic orbitals (LCAO), which are non-orthogonal. The orthogonalization leads to extended, quantummolecular orbitals which serve as a basis for CI calculation. In the Hubbard model, with $c_{i \sigma}^{+}$ $\left(c_{i \sigma}\right)$ operators creating (annihilating) electrons with spin $\sigma$ on the orbital of $i$-th dot, the Hamiltonian can be written as:

$$
\hat{H}=\sum_{\sigma, i=1}^{3} E_{i} c_{i \sigma}^{+} c_{i \sigma}+\sum_{\sigma, i, j=1, i \neq j}^{3} t_{i j} c_{i \sigma}^{+} c_{j \sigma}+\sum_{i=1}^{3} U_{i} n_{i \downarrow} n_{i \uparrow}+\frac{1}{2} \sum_{i, j=1, i \neq j}^{3} V_{i j} \varrho_{i} \varrho_{j},
$$

where $n_{i \sigma}=c_{i \sigma}^{+} c_{i \sigma}$ and $\varrho_{i}=n_{i \downarrow}+n_{i \uparrow}$ are, respectively, the spin and charge density on the $i$-th dot. The above Hamiltonian is characterized by the energy levels of the $i$-th quantum dot $E_{i}$, the tunneling matrix elements $t_{i j}$ between dots $i$ and $j$, the on-site Hubbard repulsion $U_{i}$, and the direct Coulomb matrix elements $V_{i j}$ between dots $i$ and $j$. These Hubbard parameters are schematically shown in Fig. 1(b). With one energy level per dot the triple-dot molecule can be filled with up to $N_{e}=6$ electrons.

\section{ELECTRONIC STRUCTURE OF THE TRIPLE DOT WITH 1 TO 6 ELEC- TRONS}

\section{A. One electron and one hole}

We look for the eigenenergies and eigenvectors of the Hamiltonian (11) using the exact diagonalization approach. To this end, we create all possible configurations of $N_{e}$ electrons on the three localized orbitals, write the Hamiltonian matrix in this basis, and diagonalize it numerically. In the simplest case of $N_{e}=1$ the basis contains three non-overlapping states, $\{|1\rangle,|2\rangle,|3\rangle\}$, where $|i\rangle=c_{i \downarrow}^{+}|0\rangle$ and $|0\rangle$ denotes the vacuum. In this basis the diagonal Hamiltonian matrix elements are $\langle i|\hat{H}| i\rangle=E_{i}$ and the off-diagonal elements $\langle i|\hat{H}| j\rangle=t_{i j}$. With the three dots on resonance, i.e., with $E_{1}=E_{2}=E_{3}=E$ and $t_{12}=t_{23}=t_{13}=t$, the

one-electron energy spectrum is composed of one level with energy $E_{A}=E+2 t$, and one doubly-degenerate level with energy $E_{B}=E_{C}=E-t$. The order of these levels depends 
on the sign of the element $t$. In numerical calculations of the single-particle spectrum corresponding to the potential produced by metallic gates $\frac{12}{2}$ shown in Fig. 1)(a) we find the ground state to be non-degenerate, indicating that $t<0$. Additionally, the magnitude of the tunneling matrix element can be found from the single-particle energy gap $\Delta=3|t|$.

Knowledge of the sign of the off-diagonal element allows us to construct the single-particle molecular orbitals. The ground state is $\left|M_{1}\right\rangle=\frac{1}{\sqrt{3}}(|1\rangle+|2\rangle+|3\rangle)$, while the two degenerate excited states are $\left|M_{2}\right\rangle=\frac{1}{\sqrt{2}}(|1\rangle-|2\rangle)$ and $\left|M_{3}\right\rangle=\frac{1}{\sqrt{6}}(|1\rangle+|2\rangle-2 \cdot|3\rangle)$. The states $\left|M_{2}\right\rangle$ and $\left|M_{3}\right\rangle$ were chosen to be symmetric with respect to a mirror plane passing through the dot 3 and intersecting the $(1-2)$ base of the triangle at its midpoint. However, due to the degeneracy of the two levels, any pair of orthogonal states created as linear combinations of $\left|M_{2}\right\rangle$ and $\left|M_{3}\right\rangle$ will be viable as eigenstates. The degeneracy of the excited states is a direct consequence of the symmetry of the triangular molecule. Changing its topology, e.g., by increasing the tunneling barrier between dots 1 and 3, will remove the degeneracy. In the limit of an infinite barrier, i.e., $t_{13}=0$, we deal with a linear triple-dot molecule, whose singleparticle energy spectrum consists of three equally spaced levels: $(E-\sqrt{2}|t|, E, E+\sqrt{2}|t|)$. Thus, the triangular triple dot design makes it possible to engineer the degeneracy of states solely by electrostatic means.

Now we can start to populate our triple-dot molecule with electrons. Let us start our many-body analysis with the simplest case of $N_{e}=5$. As the maximal number of electrons in our system is six, we can interpret the five-electron configurations as those of a single hole. The hole (e.g., with spin down) can be placed on either of the dots, and thus our basis consists of three configurations: $\left|1^{(H)}\right\rangle=h_{1 \downarrow}^{+}\left|N_{e}=6\right\rangle=c_{3 \uparrow}^{+} c_{2 \uparrow}^{+} c_{3 \downarrow}^{+} c_{2 \downarrow}^{+} c_{1 \downarrow}^{+}|0\rangle,\left|2^{(H)}\right\rangle=$ $h_{2 \downarrow}^{+}\left|N_{e}=6\right\rangle=c_{1 \uparrow}^{+} c_{3 \uparrow}^{+} c_{3 \downarrow}^{+} c_{2 \downarrow}^{+} c_{1 \downarrow}^{+}|0\rangle$, and $\left|3^{(H)}\right\rangle=h_{3 \downarrow}^{+}\left|N_{e}=6\right\rangle=c_{2 \uparrow}^{+} c_{1 \uparrow}^{+} c_{3 \downarrow}^{+} c_{2 \downarrow}^{+} c_{1 \downarrow}^{+}|0\rangle$, with $h_{i \sigma}^{+}$ being the creation operator of the hole with spin $\sigma$ on the $i$-th dot. It is convenient to express the energies of these configurations with respect to the total energy of the system with six electrons $E_{F}=2 E_{1}+2 E_{2}+2 E_{3}+U_{1}+U_{2}+U_{3}+4 V_{12}+4 V_{13}+4 V_{23}$. We have then $E_{1}^{(H)}=E_{F}-E_{1}-U_{1}-2 V_{12}-2 V_{13}, E_{2}^{(H)}=E_{F}-E_{2}-U_{2}-2 V_{12}-2 V_{23}$, and $E_{3}^{(H)}=E_{F}-E_{3}-U_{3}-2 V_{13}-2 V_{23}$. The three energies are respectively the diagonal terms of our single-hole Hamiltonian. The off-diagonal terms are composed out of the single-particle tunneling matrix elements. We have $\left\langle i^{(H)}|\hat{H}| j^{(H)}\right\rangle=-t_{i j}$; the negative phase is due to the anticommutation relations of the electronic creation and annihilation operators. As we can see, the single-hole Hamiltonian can be obtained from the single-electron Hamiltonian by 
appropriately modifying the diagonal terms and setting $t_{i j} \leftrightarrow-t_{i j}$. This is the signature of the particle-hole symmetry. ${ }^{27}$ However, for the triangular triple dot on resonance this symmetry is not reflected in the energy spectrum of the hole: in this case, the opposite sign of the off-diagonal element leads to a doubly-degenerate hole ground state. This property is immediately apparent in the molecular basis: we create the lowest-energy configuration by filling the molecular ground state $\left|M_{1}\right\rangle$ with two of the five electrons, and distributing the remaining three on the degenerate orbitals $\left|M_{2}\right\rangle$ and $\left|M_{3}\right\rangle$. The latter can be accomplished in two energetically equivalent ways, hence the double degeneracy. Note, however, that the electron-hole symmetry is fully restored upon transition to the linear triple-dot molecule. For this topology, the single-particle spectrum of both the electron and the hole consists of three equally spaced non-degenerate levels.

\section{B. Two electrons and two holes}

The interplay of topology and statistics is particularly important in the cases of two electrons and two holes confined in the triple dot molecule. Let us consider the case of $N_{e}=2$ first. Since the Hamiltonian (11) commutes with the total spin operator, we can classify the two-electron states into singlets and triplets. Working with the molecular basis set, we form the configuration with the lowest energy by placing both carriers with antiparallel spins on orbital $\left|M_{1}\right\rangle$. Therefore we expect the ground state of the two-electron system to be a spin singlet, irrespective of the molecule's topology. However, in order to examine the topological and statistical effects in the energy spectrum and the structure of the wave functions, we carry out a systematic analysis in the localized basis.

Due to Fermi statistics, the two electrons with parallel spins cannot occupy the same quantum dot. Hence there are only three possible triplet configurations, $\left|T_{1}\right\rangle=c_{2 \downarrow}^{+} c_{1 \downarrow}^{+}|0\rangle$, $\left|T_{2}\right\rangle=c_{3 \downarrow}^{+} c_{1 \downarrow}^{+}|0\rangle$, and $\left|T_{3}\right\rangle=c_{3 \downarrow}^{+} c_{2 \downarrow}^{+}|0\rangle$, shown schematically in Fig. 2(a). The three triplet configurations interact with each other only via the single-particle tunneling Hamiltonian. However, in evaluating the respective matrix elements we need to follow the Fermionic anticommutation rules of the creation and annihilation operators. For example, acting with $\hat{H}$ on the configuration $T_{1}$ to produce the configuration $T_{3}$ requires the evaluation of the following expression: $\hat{H}\left|T_{1}\right\rangle=+t_{31} c_{3 \downarrow}^{+} c_{1 \downarrow} c_{2 \downarrow}^{+} c_{1 \downarrow}^{+}|0\rangle$. In order to remove the electron 1 we first

have to move it around electron 2 , and so $\hat{H}\left|T_{1}\right\rangle=-t_{31} c_{3 \downarrow}^{+} c_{2 \downarrow}^{+} c_{1 \downarrow} c_{1 \downarrow}^{+}|0\rangle=-t_{31}\left|T_{3}\right\rangle$. Hence, 
tunneling of the electron from dot 1 to dot 3 in the presence of the electron in dot 2 generates an additional phase or changes the sign of the tunneling matrix element. This is of course the most elementary property of Fermions brought out so clearly in this simple model. By contrast, tunneling from dot 2 to dot 3 in the presence of electron in dot 1 does not change the sign of the tunneling matrix element. The resulting triplet Hamiltonian matrix takes the following form:

$$
\hat{H}_{T}=\left[\begin{array}{ccc}
E_{1}+E_{2}+V_{12} & t_{23} & -t_{13} \\
t_{23} & E_{1}+E_{3}+V_{13} & t_{12} \\
-t_{13} & t_{12} & E_{2}+E_{3}+V_{23}
\end{array}\right] .
$$

$\hat{H}_{T}$ is related to the one-hole Hamiltonian. This similarity becomes more apparent if $\hat{H}_{T}$ is written in the basis $\left\{\left|T_{1}\right\rangle,-\left|T_{2}\right\rangle,\left|T_{3}\right\rangle\right\}$, in which case all the off-diagonal elements acquire a negative phase. This is not surprising, since the single-hole configurations analyzed in the previous Section can be generated from the above triplet configurations simply by adding to them an inert core of three electrons spin up, one electron per dot. With the three dots on resonance and all tunneling matrix elements $t_{i j}$ equal and negative, the triplet energy spectrum is found to be $(2 E+V-|t|, 2 E+V-|t|, 2 E+V+2|t|)$. As in the case of the single hole, the lowest-energy triplet state is doubly degenerate. Moreover, the renormalization of the lowest energy $2 E+V-|t|$ from the single configuration energy $2 E+V$, as well as the gap in the triplet spectrum, are determined entirely by tunneling. The splitting between the ground and first excited states is the same as that found in the single-carrier case and equals $3|t|$.

We shall now demonstrate that topology and statistics differentiates between triplet and singlet two-electron states. The singly-occupied singlet configurations $\left|S_{1}\right\rangle,\left|S_{2}\right\rangle$, and $\left|S_{3}\right\rangle$ are obtained from the triplet configurations $\left|T_{1}\right\rangle,\left|T_{2}\right\rangle$, and $\left|T_{3}\right\rangle$ by flipping the spin of one electron and properly antisymmetrizing the configurations. For example, the configuration $\left|S_{1}\right\rangle=\frac{1}{\sqrt{2}}\left(c_{2 \downarrow}^{+} c_{1 \uparrow}^{+}+c_{1 \downarrow}^{+} c_{2 \uparrow}^{+}\right)|0\rangle$. In addition to the singly-occupied configurations there are also three doubly-occupied configurations, e.g., $\left|S_{4}\right\rangle=c_{1 \downarrow}^{+} c_{1 \uparrow}^{+}|0\rangle$, as shown in Fig. 2(b). In 
the basis of the six configurations the two-electron singlet Hamiltonian can be written as:

$$
\hat{H}_{S}=\left[\begin{array}{cccccc}
E_{1}+E_{2}+V_{12} & t_{23} & t_{13} & \sqrt{2} t_{12} & \sqrt{2} t_{12} & 0 \\
t_{23} & E_{1}+E_{3}+V_{13} & t_{12} & \sqrt{2} t_{13} & 0 & \sqrt{2} t_{13} \\
t_{13} & t_{12} & E_{2}+E_{3}+V_{23} & 0 & \sqrt{2} t_{23} & \sqrt{2} t_{23} \\
\sqrt{2} t_{12} & \sqrt{2} t_{13} & 0 & 2 E_{1}+U_{1} & 0 & 0 \\
\sqrt{2} t_{12} & 0 & \sqrt{2} t_{23} & 0 & 2 E_{2}+U_{2} & 0 \\
0 & \sqrt{2} t_{13} & \sqrt{2} t_{23} & 0 & 0 & 2 E_{3}+U_{3}
\end{array}\right] .
$$

The $3 \times 3$ upper left-hand corner of $\hat{H}_{S}$ corresponds to the three singly occupied configurations $\left|S_{1}\right\rangle,\left|S_{2}\right\rangle$, and $\left|S_{3}\right\rangle$. It is similar to the two-electron triplet Hamiltonian $\hat{H}_{T}$ but differs from it by the positive phase of the tunneling matrix element $t_{13}$. Hence, in the triangular topology of the triple-dot molecule the tunneling from dot 1 to dot 3 distinguishes between the singlet and the triplet spin configurations. By setting $t_{13}=0$, i.e., upon transition to the linear topology, this difference disappears. However, the singlet basis is still different from its triplet counterpart due to the presence of the doubly-occupied configurations.

For the dots on resonance the energies of the six singlet levels can be obtained analytically. The spectrum can be grouped into two non-degenerate levels $E_{1,2}^{S}$ :

$$
E_{1,2}^{S}=(2 E+V-2|t|)+\frac{1}{2}\left[(U-V+2|t|) \pm \sqrt{(4 \sqrt{2} t)^{2}+(U-V+2|t|)^{2}}\right]
$$

and two groups of doubly degenerate levels $E_{3-6}^{S}$ :

$$
E_{3-6}^{S}=(2 E+V+|t|)+\frac{1}{2}\left[(U-V-|t|) \pm \sqrt{(2 \sqrt{2} t)^{2}+(U-V-|t|)^{2}}\right] .
$$

In the strong coupling limit $U \gg V>|t|$ the singlet ground-state energy $E_{1}^{S} \approx(2 E+$ $V-2|t|)-\frac{8 t^{2}}{U-V}$, while the triplet energy $E_{1}^{T}=(2 E+V-|t|)$. Thus, the two-electron ground state is always a spin singlet. The singlet-triplet gap, separating $E_{1}^{S}$ from $E_{1}^{T}$ is $\Delta^{S-T} \approx|t|+\frac{8 t^{2}}{U-V}$. It is proportional to the tunneling matrix element $|t|$ and contains the second-order super-exchange correction $\sim t^{2} /(U-V)$ due to the doubly occupied singlet configurations. Removing the resonance by detuning the onsite energies $E_{i}$ enhances the contribution from the doubly-occupied states. Therefore the ground state maintains its singlet character independently of the choice of gate voltages.

The situation is qualitatively different when two holes, instead of two electrons, populate the system. The two holes are created when two electrons are removed from the closed-shell 
configuration with $N_{e}=6$, i.e., they correspond to $N_{e}=4$ electrons. In the molecular basis corresponding to the triangular triple dot we put two electrons on the lowest-energy orbital $\left|M_{1}\right\rangle$, and the remaining two electrons on the degenerate pair of orbitals $\left|M_{2}\right\rangle$ and $\left|M_{3}\right\rangle$. With this alignment of levels it is possible to create both triplet and singlet configurations, all with the same single-particle energy, and it is not immediately clear which total spin is preferred. On the other hand, in the limit of the linear triple dot the molecular orbitals are non-degenerate and the four-electron ground state is expected to be a spin singlet.

The selected two-hole singlet and triplet configurations in the localized basis are illustrated in Fig. 2(c). Let us focus on the triplets first. They involve one electron spin-up occupying the first, second, or third dot in the presence of an inert core of three spindown electrons. For example, the configuration shown in left-hand panel of Fig. 2(c) can be written as $\left|T_{1}^{(H)}\right\rangle=h_{1 \downarrow}^{+} h_{2 \downarrow}^{+}\left|N_{e}=6\right\rangle=c_{3 \uparrow}^{+} c_{3 \downarrow}^{+} c_{2 \downarrow}^{+} c_{1 \downarrow}^{+}|0\rangle$. Therefore, the hole triplet Hamiltonian is equivalent to the single-electron Hamiltonian, differing from it only in diagonal terms. For example, the energy of the configuration $\left|T_{1}^{(H)}\right\rangle$ is $\left\langle T_{1}^{(H)}|\hat{H}| T_{1}^{(H)}\right\rangle=$ $E_{F}-E_{1}-E_{2}-U_{1}-U_{2}-3 V_{12}-2 V_{13}-2 V_{23}$. The two-hole triplet Hamiltonian can also be compared to the two-electron triplet Hamiltonian $\hat{H}_{T}$, written in the modified basis set $\left\{\left|T_{1}\right\rangle,-\left|T_{2}\right\rangle,\left|T_{3}\right\rangle\right\}$ (i.e., with all off-diagonal matrix elements acquiring a negative phase). Setting aside the diagonal matrix elements, the two Hamiltonians are connected by the electron-hole symmetry transition $t_{i j} \leftrightarrow-t_{i j}$. However, unlike that of the electronic triplet, the ground state of the hole triplet is non-degenerate, and its energy is $E_{1}^{T(H)}=E_{F}-2 E-2 U-7 V-2|t|$. As it is in the case of the single electron and the single hole, the particle-hole symmetry between the two-electron triplet and the two-hole triplet is fully restored upon transition to the linear topology of the triple dot.

Let us move on to considering the two-hole singlet configurations. The singly-occupied states, illustrated in the middle panel of Fig. 2(c), involve the two holes occupying two different dots, while the doubly-occupied states, such as the one in the right-hand panel of Fig. 2(c), hold both holes on the same dot. The two-hole singlet Hamiltonian is analogous to that of the two-electron singlet, Eq. (3). However, we need to replace the energy of two-electron complexes with the energy of two-hole complexes, and change the phase of the off-diagonal elements connecting the singly-occupied configurations. The sign of elements $\sqrt{2} t_{i j}$ connecting the singly- and doubly-occupied configurations does not change, which breaks the particle-hole symmetry. 
The ground-state energy of the hole singlet for the triangular triple dot on resonance is well approximated by $E_{1}^{S(H)} \approx\left(E_{F}-2 E-2 U-7 V-|t|\right)-\frac{2 t^{2}}{U-V}$. Compared to the energy of the triplet, $E_{1}^{S(H)}$ is increased by the tunneling element $|t|$, but decreased by the super-exchange contribution $\frac{2 t^{2}}{U-V}$. Note that the two-hole super-exchange term is four times smaller than the super-exchange correction to the energy of the two-electron singlet. By increasing the tunneling or decreasing the on-site Hubbard repulsion we can increase the contribution from super-exchange and lower the energy of the singlet state. Therefore, the total spin of the two-hole ground state for the triple dot on resonance depends on the interplay of Hubbard parameters. For $2|t|<U-V$ the ground state is a spin triplet, and a triplet-singlet transition can be induced by increasing the hopping matrix element. The triplet-singlet transition can also be induced by biasing one of the dots, which lowers the energy of the doubly-occupied singlet configurations. Hence the configuration of two holes shows a nontrivial dependence on tunneling, Coulomb interactions and gate voltages allowing to control the system's magnetic moment purely by electrical means.

\section{Three electrons}

To complete our understanding of the energy levels of a triple quantum dot molecule we need to analyze the half-filled case of three electrons (or, equivalently, three holes). We start with the completely spin-polarized system, i.e., one with total spin $S=3 / 2$. In this case we can distribute the electrons on the three dots in only one way: one electron on each site, which gives a spin-polarized state $\left|a_{3 / 2}\right\rangle=c_{3 \downarrow}^{+} c_{2 \downarrow}^{+} c_{1 \downarrow}^{+}|0\rangle$. As the basis of our Hilbert space consists of one configuration only, $\left|a_{3 / 2}\right\rangle$ is the eigenstate of our system, and its energy is $E_{3 / 2}=E_{1}+E_{2}+E_{3}+V_{12}+V_{13}+V_{23}$. Let us now flip the spin of one of the electrons. This electron can be placed on any orbital, and with each specific placement the remaining two spin-down electrons can be distributed in three ways. For example, Fig. 3)(a) shows the three configurations with the spin-up electron occupying the dot 1 . Thus, altogether we can generate nine different configurations. Three of these configurations involve single occupancy of the orbitals. They can be written as $|a\rangle=c_{3 \downarrow}^{+} c_{2 \downarrow}^{+} c_{1 \uparrow}^{+}|0\rangle,|b\rangle=c_{1 \downarrow}^{+} c_{3 \downarrow}^{+} c_{2 \uparrow}^{+}|0\rangle$, and $|c\rangle=$ $c_{2 \downarrow}^{+} c_{1 \downarrow}^{+} c_{3 \uparrow}^{+}|0\rangle$. Out of these three configurations we construct the three eigenstates of the total spin operator. One of those eigenstates is $\left|a_{3 / 2}\right\rangle=\frac{1}{\sqrt{3}}(|a\rangle+|b\rangle+|c\rangle)$, and it corresponds to the

total spin $S=3 / 2$. The two other eigenstates, $\left|a_{1 / 2}\right\rangle=\frac{1}{\sqrt{2}}(|a\rangle-|b\rangle)$ and $\left|b_{1 / 2}\right\rangle=\frac{1}{\sqrt{6}}(|a\rangle+|b\rangle-$ 
$2|c\rangle)$, correspond to the total spin $S=1 / 2$. The remaining six configurations involve doublyoccupied orbitals. They are $\left|c_{1 / 2}\right\rangle=c_{2 \downarrow}^{+} c_{1 \downarrow}^{+} c_{1 \uparrow}^{+}|0\rangle,\left|d_{1 / 2}\right\rangle=c_{3 \downarrow}^{+} c_{1 \downarrow}^{+} c_{1 \uparrow}^{+}|0\rangle,\left|e_{1 / 2}\right\rangle=c_{3 \downarrow}^{+} c_{2 \downarrow}^{+} c_{2 \uparrow}^{+}|0\rangle$, $\left|f_{1 / 2}\right\rangle=c_{1 \downarrow}^{+} c_{2 \downarrow}^{+} c_{2 \uparrow}^{+}|0\rangle,\left|g_{1 / 2}\right\rangle=c_{1 \downarrow}^{+} c_{3 \downarrow}^{+} c_{3 \uparrow}^{+}|0\rangle,\left|h_{1 / 2}\right\rangle=c_{2 \downarrow}^{+} c_{3 \downarrow}^{+} c_{3 \uparrow}^{+}|0\rangle$. All these configurations are eigenstates of the total spin with $S=1 / 2$. Thus, among our nine spin-unpolarized states we have one high-spin, and eight low-spin states. In this basis the Hamiltonian matrix is block-diagonal, with the high-spin state completely decoupled. The energy corresponding to this state is equal to that of the fully polarized system discussed above, and is equal to $E_{3 / 2}$. In the basis of the nine $S=1 / 2$ configurations we construct the Hamiltonian matrix by dividing 9 configurations into three groups, each containing one of the singly-occupied configurations $|a\rangle,|b\rangle$, and $|c\rangle$. By labeling each group with the index of the spin-up electron, the Hamiltonian takes the form of a $3 \times 3$ matrix:

$$
\hat{H}_{1 / 2}=\left[\begin{array}{ccc}
\hat{H}_{1} & \hat{T}_{12} & \hat{T}_{31}^{+} \\
\hat{T}_{12}^{+} & \hat{H}_{2} & \hat{T}_{23} \\
\hat{T}_{31} & \hat{T}_{23}^{+} & \hat{H}_{3}
\end{array}\right]
$$

The diagonal matrix, e.g.,

$$
\hat{H}_{1}=\left[\begin{array}{ccc}
2 E_{1}+E_{2}+2 V_{12}+U_{1} & t_{23} & -t_{13} \\
t_{23} & 2 E_{1}+E_{3}+2 V_{13}+U_{1} & t_{12} \\
-t_{13} & t_{12} & E_{1}+E_{2}+E_{3}+V_{12}+V_{13}+V_{23}
\end{array}\right]
$$

describes the interaction of three configurations which contain spin-up electron on site 1, i.e., two doubly-occupied configurations $\left|c_{1 / 2}\right\rangle$ and $\left|d_{1 / 2}\right\rangle$, and a singly-occupied configuration $|a\rangle$ (in this order, see Fig. [3(a)). The configurations with double occupancy acquire the diagonal interaction term $U$. The three configurations involve a pair of spin-polarized electrons (spin triplet) moving on a triangular plaquette in the presence of a "spectator" spin-up electron. Because of the triplet character of the two electrons, the phase of the hopping matrix element $-t_{13}$ from site 1 to site 3 is different from the phase of the hopping matrix element $+t_{23}$ from site 2 to site 3 . As discussed above, the negative phase in $-t_{13}$ distinguishes the singlet and triplet electron pairs. The remaining matrices corresponding to spin-up electrons localized on sites 2 and 3 can be constructed in a similar fashion. The interaction between them is 
given in terms of effective hopping matrix

$$
\hat{T}_{i j}=\left[\begin{array}{ccc}
0 & -t_{i j} & 0 \\
0 & 0 & -t_{i j} \\
+t_{i j} & 0 & 0
\end{array}\right] .
$$

There is no direct interaction between the configurations with single occupancy, since such scattering process would have to involve two electrons, one with spin up and one with spin down. This cannot be accomplished by the single-particle tunneling. These states are coupled only indirectly, involving the configurations with double occupancy.

The low-energy spectrum of the Hubbard Hamiltonian of the $N_{e}=3$ quantum-dot molecule can be further approximated by the spectrum of the model spin Hamiltonian :

$$
H_{3 e}=E_{3 / 2}+\sum_{i<j} J_{i j}\left(\vec{S}_{i} \cdot \vec{S}_{j}-1 / 4\right)+\sum_{i<j<k} D_{i j k} \vec{S}_{i} \cdot\left(\vec{S}_{j} \times \vec{S}_{k}\right)
$$

Here, $E_{3 / 2}$ is the energy of the spin $S=3 / 2$ state, $J_{i j}$ are exchange matrix elements of the Heisenberg part of the spin Hamiltonian which depend on microscopic parameters of the triple dot, and $D_{i j k}$ are higher order spin-spin interactions discussed, e.g., by Scarola and Das Sarma in Ref. 33 .

We define the effective exchange constant $J$ for the triple dot molecule with three electrons in terms of the gap between the $S=1 / 2$ and $S=3 / 2$ states as $E_{3 / 2}-E_{1 / 2}=3 J / 2$. Without the higher order corrections $J$ would have been equal to the Heisenberg $J_{i j}$, otherwise it is simply related to the gap of the $N_{e}=3$ electron spectrum.

The mapping of the behavior of our system onto the effective exchange Hamiltonian (17) connects our analysis to the general formalism used in quantum computing. 10,11 Our considerations do not introduce any new elements into that formalism, but rather provide means for its realistic and accurate parametrization, reflecting the properties of an actual gated triple-dot device.

The Heisenberg Hamiltonian (7) can be used to model the behavior of three electrons confined in a triple dot treated as three coupled qubits. However, it applies also to a coded qubit scheme, in which the states of the entire molecule are treated as the logical states of a single qubit. In Ref. 12 we have presented a detailed analysis of such a system, in which we selected the two lowest total spin $1 / 2$ states as the logical states $\left|0_{L}\right\rangle$ and $\left|1_{L}\right\rangle$ of the coded qubit, respectively. In that design, the control of the energy gap between the two states by 
the gate voltage provides means for the single-qubit operations. Again, our current work allows to parametrize this model with the Hubbard parameters appropriate for a specific triple quantum dot.

\section{Comparison of Hubbard, LCAO-CI and RSP-CI results}

We shall now find the values of the Hubbard parameters appropriate for a typical triple quantum dot system. These parameters are obtained by fitting the electronic properties discussed above either to results of microscopic calculations, or to experimental data. In this Section we will focus on the former, while the latter will be discussed in Section V.

In what follows we shall express all energies in units of the effective Rydberg, $1 \mathcal{R}=$ $m^{*} e^{4} / 2 \varepsilon^{2} \hbar^{2}$, and all distances in units of the effective Bohr radius, $1 a_{B}=\varepsilon \hbar^{2} / m^{*} e^{2}$, where $e$ and $m^{*}$ are the electronic charge and effective mass, respectively, and $\varepsilon$ is the dielectric constant of the material. For GaAs parameters, $m^{*}=0.067 m_{0}$ and $\varepsilon=12.4$, we have $1 \mathcal{R}=5.93 \mathrm{meV}$ and $1 a_{B}=9.79 \mathrm{~nm}$. As the model lateral triple-dot system we take the structure shown in Fig. 1(a), discussed by us in detail elsewhere. ${ }^{12}$ We take the main gray gate to be a square with the side length of $22.4 a_{B}$. The diameter of each circular opening is $4.2 a_{B}$, the distance between the centers of each pair of the holes is $4.85 a_{B}$. The gate is positioned $14 a_{B}$ above the two-dimensional electron gas and a voltage of $-|e| V=10 \mathcal{R}$ is applied to it to create the symmetric triangular triple quantum dot.

We focus on the case of $N_{e}=3$ confined electrons. Our analysis consists of two steps. First, we find $N_{S}$ lowest-lying single-particle energies and wave functions of the system and obtain the Coulomb matrix elements involving all these states. Second, we calculate the three-electron eigenenergies within the configuration-interaction (CI) approach.

The one-electron properties of the system can be derived in a real-space approach (RSP) involving numerical diagonalization of the discretized single-particle Hamiltonian. 12 We compute $N_{S}=9$ lowest-lying levels. The ground state is separated from the first excited state by an energy gap of $0.1877 \mathcal{R}$, while the gap between the first and second excited states is much smaller and equal to $0.0061 \mathcal{R}$. This agrees well with the Hubbard model, predicting a degeneracy of the two excited states. Also, from the average gap between the ground and excited states, which in the Hubbard model equals $3|t|$, we can extract the tunneling parameter $t_{12}=t_{13}=t_{23}=-0.0636 \mathcal{R}$. 
The RSP approach, while being accurate, is computationally intensive. As an alternative we consider a method based on the linear combination of atomic orbitals (LCAO) $\underline{\underline{38}}$ To this end, we approximate the numerical triple-dot lateral confinement, obtained as a solution of the Poisson equation, with a sum of three Gaussians:

$$
V(x, y)=-\sum_{i=1}^{3} V_{0}^{(i)} \exp \left(-\frac{\left(x-x_{i}\right)^{2}+\left(y-y_{i}\right)^{2}}{d_{i}^{2}}\right) .
$$

The pairs $\left(x_{i}, y_{i}\right)$ are coordinates of the center of each dot. For our symmetric triple dot a good fit is obtained for $d_{1}=d_{2}=d_{3}=2.324 a_{B}$ and $V_{0}^{(1)}=V_{0}^{(2)}=V_{0}^{(3)}=5.864 \mathcal{R}$. We seek the quantum-molecular single-particle states in the form of linear combinations of single-dot orbitals localized on each dot. To simplify the calculations, we take these orbitals to be harmonic-oscillator (HO) wave functions of a two-dimensional parabolic potential, obtained by extracting the second-order component from each Gaussian. We take one stype $\mathrm{HO}$ orbital per dot, and solve the generalized single-particle eigenproblem formulated in this nonorthogonal basis set. $\stackrel{38}{\underline{3}}$ As a result, we obtain $N_{S}=3$ quantum-molecular levels: a non-degenerate ground state and a doubly degenerate excited state, separated by a gap of $0.0354 \mathcal{R}$. This structure of levels is reproduced by the Hubbard model with the tunneling parameter $t=-0.0118 \mathcal{R}$. Note that in the LCAO case the tunneling gap is much smaller than that obtained in the RSP calculation. This is due to the restricted LCAO basis set, which underestimates the overlap between the single-dot orbitals. The agreement between the two approaches can be improved upon inclusion of the $p$ and $d$ HO orbitals in the LCAO basis, at the expense of clarity $\underline{\underline{38}}$

With the single-particle energies and the Coulomb matrix elements calculated using the quantum-molecular orbitals, we can now proceed to the CI calculation of three-electron properties. We create all possible configurations of the three electrons with total $S_{z}=-1 / 2$ on $N_{S}$ quantum-molecular states ( $N_{S}=9$ for the RSP, and $N_{S}=3$ for the LCAO approach), build the many-body Hamiltonian matrix in the basis of these configurations, and diagonalize it numerically ${ }^{\underline{39}}$ The resulting spectra are shown in the left-hand parts of Fig. 3(b) for the RSP-CI, and in Fig 3(c) for the LCAO-CI approach. From the gaps separating the levels we can extract the Hubbard interaction parameters. They are: $V=0.479 \mathcal{R}$ and $U=1.539 \mathcal{R}$ in the RSP-CI case, and $V=0.422 \mathcal{R}$ and $U=2.557 \mathcal{R}$ in the LCAO-CI case. The resulting Hubbard three-electron spectra are plotted in the right-hand parts of the Figures 3(b) and (c). 
All models predict the ground state of the system to be doubly degenerate and to have total spin $S=1 / 2$. This is easily understood by building the lowest-energy configuration with triple-dot molecular orbitals: two out of three electrons are placed on the orbital $\left|M_{1}\right\rangle$ with antiparallel spins, and the third electron - on one of the degenerate orbitals $\left|M_{2}\right\rangle$ or $\left|M_{3}\right\rangle$. This configuration has total spin $S=1 / 2$, and no spin transition is expected upon the change of the system's topology. Further, in all spectra the first excited state has total spin $S=3 / 2$. This is the $\left|a_{3 / 2}\right\rangle$ state from the previous Section, equivalent to the spinpolarized configuration with one electron per dot. The energy gap between the low-spin and the high-spin states, expressed in the language of the effective Heisenberg Hamiltonian, is equal to $3 J / 2$, with $J>0$ (the ground state is antiferromagnetic).

The remaining excited states, involving doubly occupied configurations, are visible at higher energies. They are separated from the low-energy, singly-occupied states by a gap proportional to the Hubbard onsite interaction parameter $U$. This parameter is larger in the LCAO-CI approach because of the relatively small spatial extent of the HO basis states. This is consistent with the underestimated tunneling gap found earlier in the calculation of the single-particle spectra. On the other hand, the Hubbard interdot interaction parameter $V$ is similar in both approaches. In the Hubbard model, the high-energy part of the spectrum is composed of three doubly-degenerate states. The degeneracy of the lowest and the highest level within this band is well reproduced in both microscopic models, while the middle level appears to be split by a smaller gap in the LCAO-CI, and a larger gap in the RSP-CI spectrum. Finally, the RSP-CI result reveals further levels, with both total spin $S=1 / 2$ and $S=3 / 2$. Their appearance is a consequence of the extended basis, containing $N_{S}=9$ single-particle molecular states, compared to $N_{S}=3$ states in LCAO-CI and Hubbard models. The additional states can be accounted for systematically by including more than one orbital per dot in the localized basis set. $\underline{38}$ To conclude this analysis, we find the Hubbard model to give qualitatively correct results but caution has to be exercised when making a quantitative comparison.

Up to now we have explored the case of a symmetric triangular triple-dot molecule. Let us now tune the topology of the system using the gate $G V_{13}$. Figure 4 (a) shows the threeelectron spectrum obtained with the RSP-CI method as a function of the voltage applied to this gate, and the low-energy part of this spectrum is shown in the inset to this Figure. 12 In the Hubbard model, this change of topology can be accounted for by tuning the single- 
particle tunneling parameter $t_{13}$. The corresponding spectra are shown in Fig. 4(b). Both approaches indicate that the change of topology of the molecule leads to a splitting of the two degenerate $S=1 / 2$ levels. This property is a consequence of the removal of degeneracy of the single-particle molecular orbitals: with two electrons forming a spin singlet on the orbital $\left|M_{1}\right\rangle$, the third electron probes the splitting between the levels $\left|M_{2}\right\rangle$ and $\left|M_{3}\right\rangle$ The ability to tune the splitting by electrostatic means only suggests a possible use of the two low-spin states as logical states of a voltage-controlled coded qubit. $\frac{12}{1 n}$ the language of Hubbard configurations discussed in the previous Section, these states can be written as $\left|0_{L}\right\rangle=\alpha_{0} \frac{1}{\sqrt{2}}(|a\rangle-|b\rangle)+\beta_{0}\left|\Delta_{0}\right\rangle$ and $\left|1_{L}\right\rangle=\alpha_{1} \frac{1}{\sqrt{6}}(|a\rangle+|b\rangle-2|c\rangle)+\beta_{1}\left|\Delta_{1}\right\rangle$, where $\left|\Delta_{0}\right\rangle,\left|\Delta_{1}\right\rangle$ are contributions of the doubly-occupied configurations.

\section{CHARGING DIAGRAM OF THE TRIPLE DOT}

We can now construct the charging diagram of the triple-dot molecule. For any number of electrons $N_{e}$ (1 to 6) and any pair of gate voltages, or equivalently, quantum dot energies $E_{i}$, we can establish the ground-state energy $E_{G S}\left(N_{e}\right)$ by diagonalizing the Hubbard Hamiltonian. We use these energies to calculate the chemical potential of the triple quantum dot molecule $\mu\left(N_{e}\right)=E_{G S}\left(N_{e}+1\right)-E_{G S}\left(N_{e}\right)$. When $\mu\left(N_{e}\right)$ equals the chemical potential $\mu_{L}$ of the leads, the $N_{e}+1$ st electron is added to the $N_{e}$-electron quantum-dot molecule. This establishes the total number of electrons $N_{e}$ in the quantum dot molecule and their total spin as a function of applied voltages, or quantum-dot energies. Changes in electron numbers can be detected by the Coulomb blockade (CB), spin blockade, or charging spectroscopies. $\underline{4}, \underline{8}$ The calculated stability diagram, with Hubbard parameters extracted from the RSP-CI calculation for three electrons, is shown in Fig. 5 ,

Figure 5(a) shows the addition spectrum for the triple dot on resonance, i.e., when all dots are characterized by the same onsite energies, tunneling amplitudes, and Coulomb matrix elements. We follow the addition spectrum as we change the onsite energy $E$ of each quantum dot with respect to the chemical potential of the leads $\mu_{L}=0$. From the condition $\mu_{L}=E-2|t|$, the energy $E(1)$ corresponding to the addition of the first electron equals twice the hopping matrix element, $E(1)=2|t|$. At this energy the first Coulomb blockade peak of the triple quantum dot molecule should be observed.

The onsite energy corresponding to the second CB peak, i.e., when the second electron 
enters the dot, equals $E(2)=-V+\frac{8 t^{2}}{U-V}$. The energy to add the second electron, or the spacing between the first two peaks $\Delta_{12}=V+2|t|-\frac{8 t^{2}}{U-V}$, is proportional to the direct Coulomb interaction $V$ between two electrons on two different dots and to twice the tunneling matrix element, and is reduced by super-exchange interaction.

The third electron enters the molecule for $E(3)=-2 V+\frac{3 J}{2}-2|t|-\frac{8 t^{2}}{U-V}$ and the spacing of the third and second CB peak equals $\Delta_{23}=V+2|t|-\frac{3 J}{2}+2 \frac{8 t^{2}}{U-V}$. This spacing is proportional to $V, 2 t$, and twice the super-exchange, but is reduced by the spin gap of the $N_{e}=3$ electron complex, equal to $3 J / 2$. The difference between the spacing of the $(2,1)$ and $(3,2)$ peaks, $\Delta_{23}-\Delta_{12}=-\frac{3 J}{2}+3 \frac{8 t^{2}}{U-V}$, directly measures the difference between the exchange in the triply occupied quantum dot molecule and three times the super-exchange in a doubly occupied quantum dot molecule.

The half-filled molecule can also be probed by adding the fourth electron. This electron enters the dot for $E(4)=-U-2 V-\frac{3 J}{2}+2|t|$. Since the four-electron states are the first to be built by doubly-occupied configurations, the corresponding CB peak is spaced from the one for the third electron by a large on-site Coulomb energy $U$. The separation between CB peaks equals $\Delta_{43}=U+2 \frac{3 J}{2}-4|t|-\frac{8 t^{2}}{U-V}$. It reflects the triplet state of two holes, and is a measure of $U, J$, and $|t|$ but not directly $V$.

The expressions for peak spacings can be used to extract the Hubbard parameters of the system from the measured CB spectrum of the triple dot on resonance. With these parameters we can now explore the full charging diagram of the molecule as a function of the onsite dot energies. If each of the energies $E_{i}$ can be varied independently, the resulting stability diagram is three-dimensional, and therefore difficult to visualize. This is why in the proposed device, shown in Fig. 1(a), the dots one and two are tuned by a single gate $V G_{1}$ while dot three is tuned by gate $V G_{3}$. Figure 5(b) shows the corresponding cross-section of the stability diagram, calculated with the RSP-CI Hubbard parameters. The diagram shows the regions $\left(E_{1}=E_{2}, E_{3}\right)$ where different electron numbers are stable. The regions are denoted by $\left(N_{1}, N_{2}, N_{3}\right)$ where $N_{i}$ is the number of electrons (for $N_{e} \leq 3$ ) or holes (for $\left.N_{e}>3\right)$ in the $i$-th dot. For example, $(1,1,1)$ denotes the half-filled triple dot with one electron in each dot. Additionally, the regions are color-coded to indicate the total spin of the molecule. We find that the two electrons always form a spin singlet, but the total spin of the two-hole system can be changed from the triplet, which is stable close to the resonance condition, to a singlet. This transition can be induced by tuning the gate voltages and does 
not require the presence of a magnetic field.

\section{COMPARISON OF THEORETICAL AND EXPERIMENTAL CHARGING DI- AGRAMS}

We now turn to the comparison of theory with experiment. The addition diagram of a triple-dot lateral device, measured recently by our group, $\underline{\underline{8}}$ is shown in Fig. 6(a). The layout of the metallic gates composing the device and the resulting potential minima are shown in the inset to this Figure. A similar arrangement of five bottom gates and one top gate has been used to define electrostatically the lateral double quantum dot, with the quantum point contact (QPC) used as a charge detector $, \underline{4}, \underline{5}, \underline{6}$ To this end, a sufficiently large negative voltage is applied to the gates $1 \mathrm{~B}$ and $5 \mathrm{~B}$, as well as to the top gate $(\mathrm{T})$ and the middle bottom gate (the gate $3 \mathrm{~B}$ ). The smaller bottom gates, $2 \mathrm{~B}$ and $4 \mathrm{~B}$, are then used as plungers, i.e., tuned with a smaller voltage to influence each of the dots locally. However, if a large negative voltage is applied to the small gates $2 \mathrm{~B}$ and $4 \mathrm{~B}$, and a smaller voltage - to the middle gate 3B, a structure of three potential minima is created: the dot 1 close to the gate $1 \mathrm{~B}$, the dot 3 - near the gate $5 \mathrm{~B}$, and the dot 2 - in the middle, between the gates $\mathrm{T}$ and $3 \mathrm{~B}$. In this arrangement, the dots form a linear chain, so the electrons cannot tunnel from the surrounding 2DEG directly to the middle dot. In the experimental addition spectrum, however, three sets of lines with distinct slopes are detected, indicating that each dot is connected to the leads independently. Moreover, two sets of lines show a stronger dependence on the voltage $V_{1 B}$ than on $V_{5 B}$, while one set exhibits an opposite tendency. This suggests a formation of a ring-type arrangement, consisting of two dots contained on the left-hand side, and one - on the right-hand side of the device, as shown schematically in the inset to Fig. 6(a). The double potential minimum on the left-hand side is created most

likely by a mesoscopic fluctuation of the background potential of the sample. This makes it difficult to control the dots 1 and 2 independently. On the other hand, the proposed sample layout, shown in Fig. 1(a), results in the formation of electrostatically defined triangular triple-dot confinement. The design has been adapted to approximate the functionality of the experimental device, but it can be modified to allow for independent control of both onsite and tunneling energies, however at the expense of a more difficult to fabricate, vertical structure of multiple gates. This is why we do not suggest this sample layout as a practical 
gating scheme, but use it to demonstrate the degree of control we aim to achieve in our future designs of triple-dot systems.

In our experiment, the energy landscape of the lateral confinement is tuned by all six gates, but the addition diagram is measured only as a function of two gate voltages, $V_{1 B}$ and $V_{5 B}$. When these voltages are set to large negative values, the system is completely depleted of electrons. This corresponds to the bottom left-hand region $(0,0,0)$ of the stability diagram. In the Hubbard model this region would correspond to the onsite dot energies $E_{i}$ being larger than the chemical potential of the leads, i.e., the upper right-hand corner $(0,0,0)$ of the diagram in Fig. 5(b). As the gate voltages are made less negative, the energies $E_{i}$ are lowered, and subsequent electrons enter the molecule. These addition events are detected in the QPC current $I_{Q P C}$, reacting to the changes in the charge distribution in the system. The dark lines in Fig. 6(a) denote the boundaries between regions corresponding to different stable electron numbers. Let us focus on the addition line composed of sections $\mathrm{A}$, B, and C, which marks the addition of the first electron to dot 1,2, or 3, respectively. The quantum molecular character of the system is revealed by the curvature of this line close to the regions denoted as $\mathrm{D}$ and $\mathrm{E}$, where the dots 1 and 2, or 2 and 3 are on resonance, respectively. It is clear that the dots 1 and 2 are coupled much more strongly than the dots 2 and 3 . The dashed lines drawn in these regions connect the points of inflection of the addition lines of the first and second electron. In the absence of quantum tunneling, these lines would correspond to the conditions $E_{1}=E_{2}$ and $E_{2}=E_{3}$, respectively. Away from these regions the dots are far from resonance, and the electrons are added to orbitals well-localized on individual dots. Therefore, the asymptotes drawn with respect to the sections $\mathrm{A}, \mathrm{B}$, and $\mathrm{C}$ of the one-electron line will define the respective single-dot properties.

As a first approximation the onsite energies $E_{i}$ are expressed as linear functions of the two gate voltages:

$$
E_{i}=\alpha_{i} V_{1 B}+\beta_{i} V_{5 B}+\gamma_{i}
$$

Let us first focus on establishing the coefficients $\alpha_{i}$ and $\beta_{i}$. In general, we seek six coefficients, but have only five equations at our disposal (the asymptotes to sections A, B, and $\mathrm{C}$, and the two resonance conditions $\mathrm{D}$ and $\mathrm{E}$ ), so at least one parameter has to be established independently. In this case, however, from independent measurements we know three coefficients: $\alpha_{1}=\alpha_{2}=-100 \mathrm{meV} / \mathrm{V}$, and $\beta_{3}=-100 \mathrm{meV} / \mathrm{V}$. The equality of the coefficients $\alpha_{1}$ and $\alpha_{2}$ is reflected by the vertical character of the line $\mathrm{D}$. The remaining three 
coefficients can now be easily extracted from the asymptotes to the sections A, B, and C, and are $\beta_{1}=-19.0 \mathrm{meV} / \mathrm{V}, \beta_{2}=-26.923 \mathrm{meV} / \mathrm{V}$, and $\alpha_{3}=-22.923 \mathrm{meV} / \mathrm{V}$.

We are now in a position to convert the charging diagram from the coordinates $\left(V_{1 B}, V_{5 B}\right)$ to the energy coordinates, assuming for the moment that the coefficients $\gamma_{i}$ are zero. Since the Hubbard parameters are of one-dot and two-dot character only, they can be systematically fitted by extracting the features involving the dot pair $(i, j)$ from the charging diagram and replotting them as a function of the energies $\left(E_{i}, E_{j}\right)$. As an example we discuss the case of $i=1, j=2$, with the translated charging diagram shown in Fig. 6(b). In this Figure, the solid black lines show the experimental data, while the dotted horizontal and vertical lines are the asymptotes. As already discussed, in the upper right-hand region, corresponding to large values of $E_{1}$ and $E_{2}$, the system is empty. Starting in this region, we can decrease the energy $E_{1}$ while maintaining $E_{2}$ constant: this corresponds to moving horizontally across the diagram. Along the way we shall first cross the rightmost vertical asymptote, which will mark the addition of the first electron to dot 1 in the zero-coupling regime (i.e., $\left.t_{12}=0\right)$, thereby driving the system into the configuration $(1,0,0)$. Because of our assumption of the chemical potential of the leads $\mu_{L}=0$, the energy $E_{1}$, which this asymptote defines, is equal simply to $-\gamma_{1}$. In the similar fashion, from the region $(0,0,0)$ we can move vertically downwards, decreasing $E_{2}$ while keeping $E_{1}$ constant. Crossing of the top horizontal asymptote marks the addition of the first electron into the second dot, i.e., formation of a configuration $(0,1,0)$, and defines the parameter $\gamma_{2}$. The two asymptotes cross at a right angle, which would be an expected behavior of the addition lines at zero coupling. However, the experimental data trace a hyperbola, whose curvature is a direct measure of the single-particle tunneling element $t_{12}$.

Let us now position ourselves in the region in which the first electron has entered the dot 1 (the region $(1,0,0)$, the top part of the diagram). As we move vertically downwards, we encounter the top horizontal asymptote. This line would mark the addition of the second electron, and its placement on the second dot, but only in the case the electrons were not interacting. Since it is necessary to compensate for the Coulomb off-site charging energy, the actual addition takes place at lower energy $E_{2}$, i.e., upon crossing of the horizontal asymptote second from the top. The energy distance between the two horizontal asymptotes corresponds directly to the Hubbard parameter $V_{12}$. An identical value is obtained by performing an analogous analysis starting in the region $(0,1,0)$ (the right-hand edge of the 
diagram), and moving horizontally to the left. Finally, we can find the onsite Coulomb terms $U_{1}$ and $U_{2}$ by examining the energy differences between asymptotes marking the addition of the second and the third electrons. These terms define the size of the stability region $(1,1,0)$, as shown in Fig. 6(b). By using a similar analysis for the features involving the second and third dots, and then the first and third dots, we can systematically extract all Hubbard parameters. In our case they are (in meV): $\gamma_{1}=-34.238, \gamma_{2}=-37.169, \gamma_{3}=-36.246$, $t_{12}=-0.053, t_{13}=t_{23}=-0.0077, V_{12}=0.4623, V_{13}=0.0448, V_{23}=0.0962, U_{1}=2.238$, $U_{2}=2.1262$, and $U_{3}=1.8923$. Figure [7(a) shows the charging diagram computed with the Hubbard model with the above parameters as a function of the gate voltages. It coincides exactly with the experimental diagram shown in Fig. 6(a).

Note that with the dependence of the onsite energies $E_{i}$ on gate voltages defined in Eq. (9), the triple-dot molecule is on resonance only for $\left(V_{1}, V_{5}\right)=(-0.363 V,-0.37 \mathrm{~V})$. In Fig. 6(a) this point is found in the $(0,0,0)$ region, and this is why the charging diagram is essentially a superposition of two double-dot diagrams, and no features unique to the resonant triple-dot molecule are visible. It has been demonstrated $\underline{8}$ that by retuning the gates making up the device the point of resonance can be shifted to the region of the diagram where the electrons start populating the system. This results in the appearance of the quadruple points, in which four different electronic configurations are on resonance, and charge redistribution effects similar to those in quantum cellular automata.

Now let us assume that we can control the three quantum dot energies in our device independently, with the dots one and two on resonance. Using the Hubbard parameters found for our device we compute the charging diagram as a function of $E_{1}=E_{2}, E_{3}$, and shown it in Fig. 7(b). The computed charging diagram is similar to that in Fig. 5(b), in which we resolve the spins of the electronic states. The results agree with our theoretical predictions, including the existence of the triplet four-electron phase. We find this phase stable across only a very small range of onsite energies. Detecting the electrostatically driven triplet-singlet transition will be investigated in the future.

\section{CONCLUSIONS}

In conclusion, we presented a theory of electronic properties of a triple quantum dot molecule. The electronic properties can be understood in terms of a topological Hunds rule, 
which determines the spin of the molecule as a function of the filling of the electronic shell.

When the three dots form a symmetric triangular molecule on resonance, the ground state for two electrons is a spin singlet, for three electrons (half-filled shell) it is an antiferromagnetic $S=1 / 2$ configuration, and for two holes it is a triplet. The topology and statistics enter through the dependence of the energies of states on total spin. For example, the singlet-triplet splitting is found to depend on tunneling and not on charge. The energetics and the charging diagram are mapped out, compared with experiment and analyzed in detail.

We have also demonstrated that the Hubbard model is capable of reproducing the experimental addition spectra in a quantitative manner. We have described a systematic procedure of extracting the Hubbard parameters from the elements of the measured charging diagram. Since in the experiment the single-particle orbital energies are controlled by gate voltages, it should be possible to induce the triplet-singlet transition for a four-electron molecule purely by electrostatic means. Our preliminary calculations indicate that such a transition should be possible in the case of the experimental lateral triple-dot device used by Gaudreau et al. . $^{-}$ Future work, including requirements for new device layout, is outlined.

1 M. Ciorga, A. S. Sachrajda, P. Hawrylak, C. Gould, P. Zawadzki, S. Jullian, Y. Feng, and Z. Wasilewski, Phys. Rev. B 61, 16315 (2000).

2 S. Tarucha, D. G. Austing, T. Honda, R. J. van der Haage, and L. P. Kouwenhoven, Phys. Rev. Lett. 77, 3613 (1996).

3 A. W. Holleitner, R. H. Blick, A. K. Hüttel, K. Eberl, and J. P. Kotthaus, Science 297, 70 (2002).

4 M. Pioro-Ladriere, R. Abolfath, P. Zawadzki, J. Lapointe, S. Studenikin, A. S. Sachrajda, and P. Hawrylak, Phys. Rev. B 72, 125307 (2005).

5 F. H. Koppens, J. A. Folk, J. M. Elzerman, R. Hanson, L. H. W. van Beveren, I. T. Vink, H. P. Tranitz, W. Wegscheider, L. P. Kouwenhoven, and L. M. K. Vandersypen, Science 309, 1346 (2005).

6 J. R. Petta, A. C. Johnson, J. M. Taylor, E. A. Laird, A. Yacoby, M. D. Lukin, C. M. Marcus, M. P. Hanson, and A. C. Gossard, Science 309, 2180 (2005).

7 T. Hatano, M. Stopa, and S. Tarucha, Science 309, 268 (2005). 
8 L. Gaudreau, S. Studenikin, A. Sachrajda, P. Zawadzki, A. Kam, J. Lapointe, M. Korkusinski, and P. Hawrylak, Phys. Rev. Lett. 97, 036807 (2006).

9 J. A. Brum and P. Hawrylak, Superlattices Microstruct. 22, 431 (1997).

10 D. Loss and D. P. DiVincenzo, Phys. Rev. A 57, 120 (1998).

11 D. P. DiVincenzo, D. Bacon, J. Kempe, G. Burkard, and K. B. Whaley, Nature 408, 339 (2000).

12 P. Hawrylak and M. Korkusinski, Solid State Commun. 136, 508 (2005).

13 Y. S. Weinstein, S. C. Hellberg, and J. Levy, Phys. Rev. A 72, 020304 (2005).

14 V. W. Scarola, K. Park, and S. Das Sarma, Phys. Rev. Lett. 93, 120503 (2004).

15 D. S. Saraga and D. Loss, Phys. Rev. Lett. 90, 166803 (2003).

16 K. Le Hur, P. Recher, E. Dupont, and D. Loss, Phys. Rev. Lett. 96, 106803 (2006).

17 M. Stopa, Phys. Rev. Lett. 88, 146802 (2002).

18 A. Vidan, R. M. Westervelt, M. Stopa, M. Hanson, and A. C. Gossard, Appl. Phys. Lett. 85, 3602 (2004).

19 K. Ingersent, A. W. W. Ludwig, and I. Affleck, Phys. Rev. Lett. 95, 257204 (2005).

20 R. Žitko and J. Bonča, Phys. Rev. B 74, 045312 (2006).

21 R. Žitko, J. Bonča, A. Ramšak, and T. Rejec, Phys. Rev. B 73, 153307 (2006).

22 Z.-T. Jiang, Q.-F. Sun, and Y. Wang, Phys. Rev. B 72, 045332 (2005).

23 T. Kuzmenko, K. Kikoin, and Y. Avishai, Phys. Rev. Lett. 96, 046601 (2006).

24 T. Kuzmenko, K. Kikoin, and Y. Avishai, Phys. Rev. B 73, 235310 (2006).

25 Y. Avishai, T. Kuzmenko, and K. Kikoin, Physica E 29, 334 (2005).

26 R. Sakano and N. Kawakami, Phys. Rev. B 72, 085303 (2005).

27 M. W. Long and R. Fechrenbacher, J. Phys.: Condens. Matter 2, 10343 (1990).

28 I. S. Elfimov, S. Yunoki, and G. A. Sawatzky, Phys. Rev. Lett. 89, 216403 (2002).

29 P. W. Anderson, P. A. Lee, M. Randeria, T. M. Rice, N. Trivedi, and F. C. Zhang, J. Phys.: Condens. Matter 16, R755 (2004).

30 N. Doiron-Leyraud, I. R. Walker, L. Taillefer, M. Steiner, S. R. Julian, and G. G. Lonzarich, Nature 425, 595 (2003).

31 E. Saitoh, S. Okamoto, K. T. Takahashi, K. Tobe, K. Yamamoto, T. Kimura, S. Ishihara, S. Maekawa, and Y. Tokura, Nature 410, 180 (2001).

32 M. Coey, Nature 430, 155 (2004).

33 V. W. Scarola and S. D. Sarma, Phys. Rev. A 71, 032340 (2005). 
34 A. Mizel and D. A. Lidar, Phys. Rev. Lett 92, 077903 (2004).

35 A. Mizel and D. A. Lidar, Phys. Rev. B 70, 115310 (2004).

36 R. Woodworth, A. Mizel, and D. A. Lidar, J. Phys.: Condens. Matter 18, S721 (2006).

37 M. L. Ladrón de Guevara and P. A. Orellana, Phys. Rev. B 73, 205303 (2006).

38 I. Puerto-Gimenez, M. Korkusinski, and P. Hawrylak, unpublished.

39 A. Wensauer, M. Korkusinski, and P. Hawrylak, Solid State Commun. 130, 115 (2004). 
FIG. 1: (Color online) (a) Cross-sectional view of a model of the three coupled gated lateral quantum dots. The grey rectangular gate contains three circular openings, which translate into minima of the electrostatic potential at the level of the two-dimensional electron gas. The red and green gates can be used to shift the potential minima of the dots underneath them with respect to the rest of the system. The blue gate is used to tune the tunneling barrier between dots 1 and 3 . (b) Schematic representation of the triple dot structure.

FIG. 2: (Color online) The three triplet configurations in a two-electron triple dot molecule (a) and examples of singly and doubly occupied singlet configurations for two electrons (b) and two holes (c).

FIG. 3: (Color online) (a) Configurations of the three-electron quantum dot with one spin-up electron occupying dot 1. (b) Three-electron energy levels calculated using the RSP-CI approach to the device shown in Fig. 1 (left-hand part) and using the appropriately fitted Hubbard model (right-hand part). (c) Similar spectra obtained with the LCAO-CI method 
FIG. 4: (Color online) (a) Energy levels calculated by RSP-CI technique, measured from the ground state, as a function of the voltage applied to the control gate $V G_{13}$. Black lines show energies of total-spin-1/2 states, the red line shows the energy of the spin- $3 / 2$ state. Inset shows the three lowest energies as a function of the gate voltage. (b) Energies of three electrons localized on three Hubbard sites as a function of the tunneling amplitude $t_{13}$ measured from the ground state. Hubbard model parameters were extracted from exact diagonalization results.

FIG. 5: (Color online) (a) Charging diagram of the triple quantum dot on resonance as a function of energy level $E$ of each dot from $N_{e}=0$ to $N_{e}=6$ electrons. (b) Stability diagram $\left(E_{1}-\mu=\right.$ $\left.E_{2}-\mu ; E_{3}-\mu\right)$ of the triple dot molecules with dots 1 and 2 tuned by a common gate. $\left(N_{1}, N_{2}, N_{3}\right)$ denotes average electron occupation and $\left(-N_{1},-N_{2},-N_{3}\right)$ denotes average hole occupation of each dot. $\mu$ denotes the chemical potential of the leads. The light gray, yellow, and brown colors mark the stability regions of phases with total spin $0,1 / 2$, and 1 , respectively.

FIG. 6: (a) (Color online) Addition spectrum of a lateral triple quantum dot molecule measured

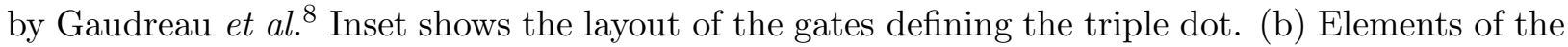
triple-dot addition spectrum involving dots 1 and 2 only, drawn as a function of onsite energies of the two dots. The Hubbard parameters can be extracted directly from this diagram (see text for details).

FIG. 7: (a) Addition spectrum of the lateral triple-dot device calculated within the Hubbard model after fitting to the experimental spectra shown in Fig. 6(a). (b) (Color online) The same spectrum shown in the form of a charging diagram as a function of the single-dot onsite energies. Light gray, yellow, and brown color marks the stability region of molecules with total spin $0,1 / 2$, and 1 , respectively. 


(a)
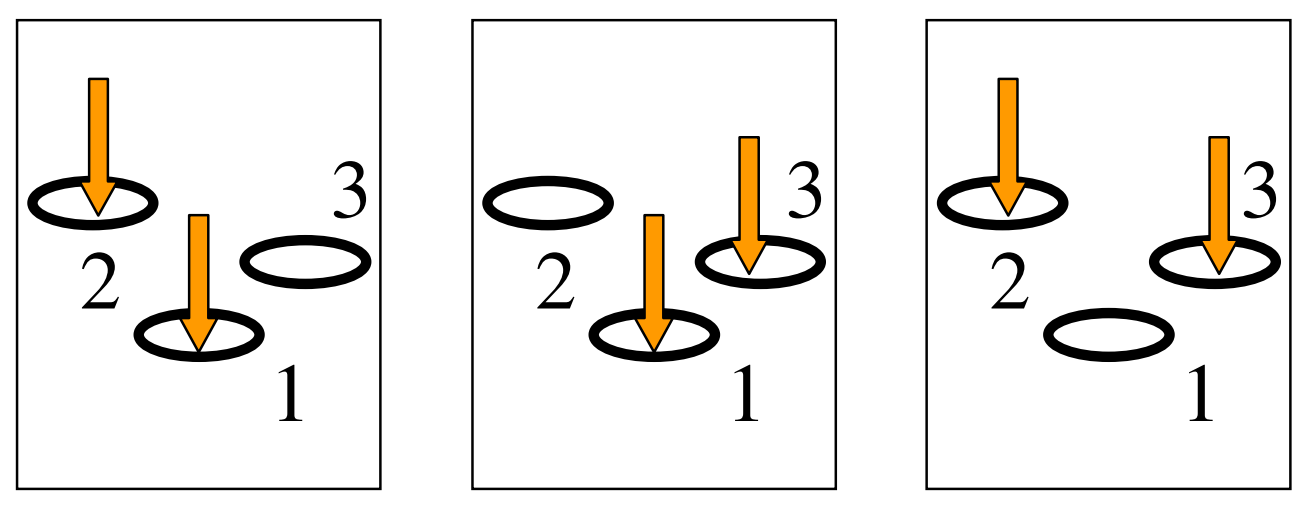

(b)
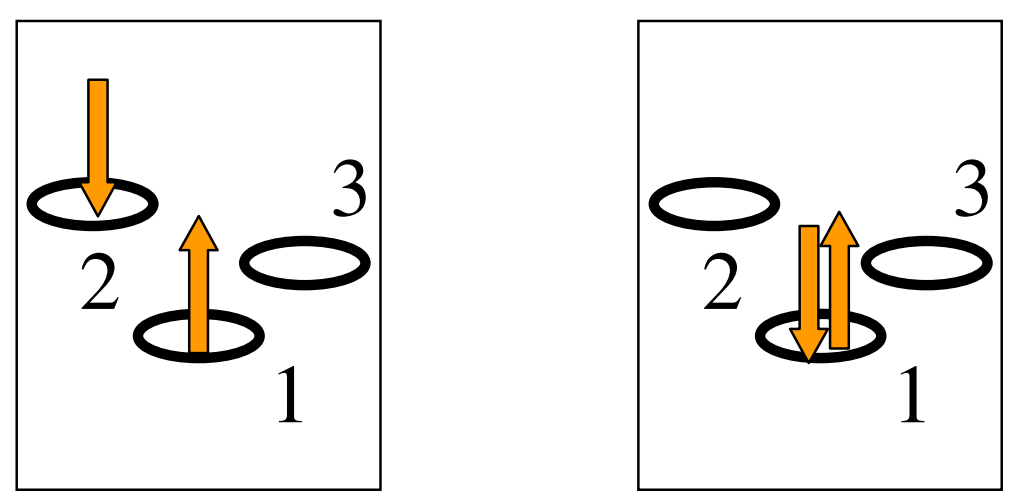

(c)


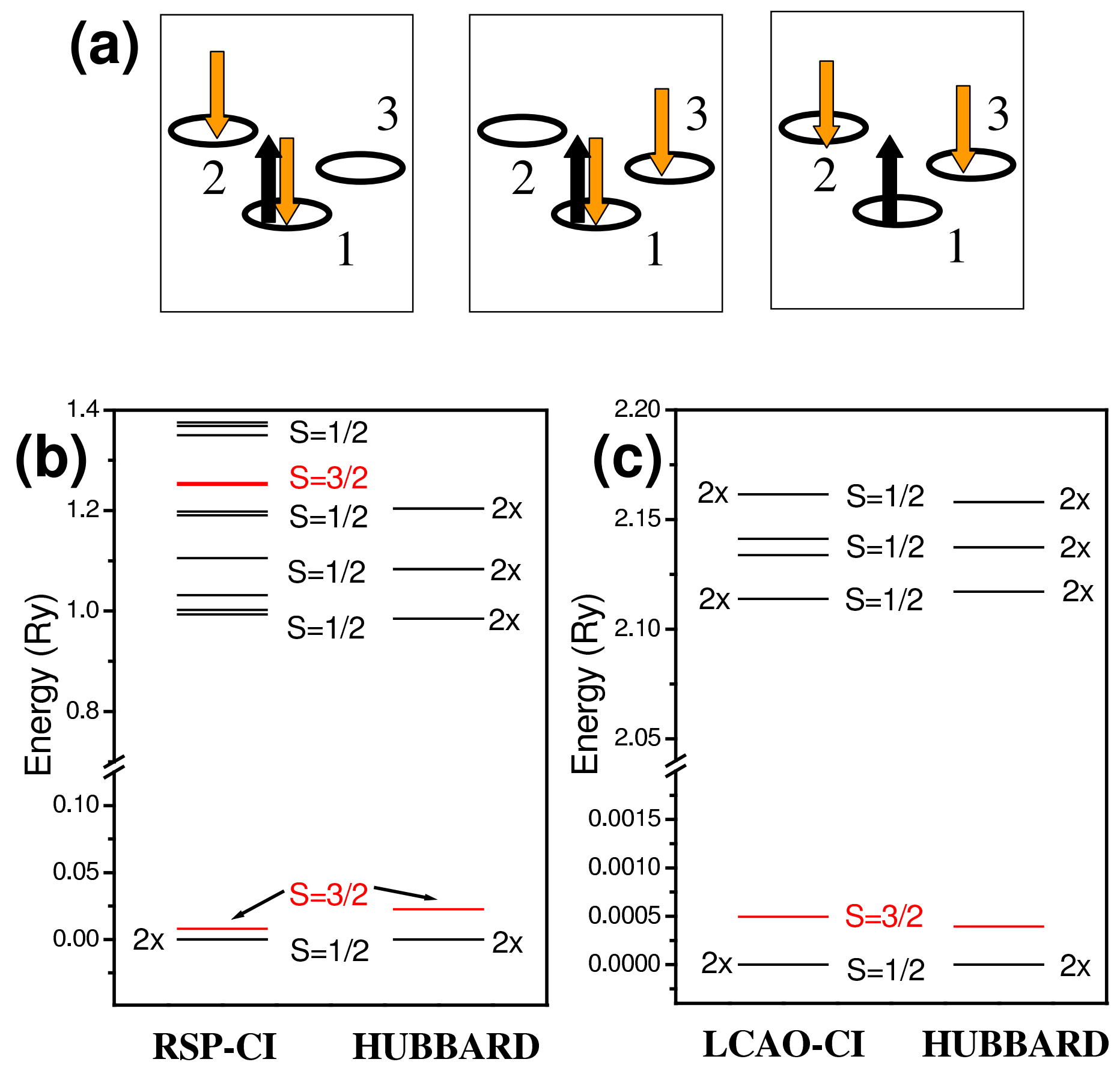




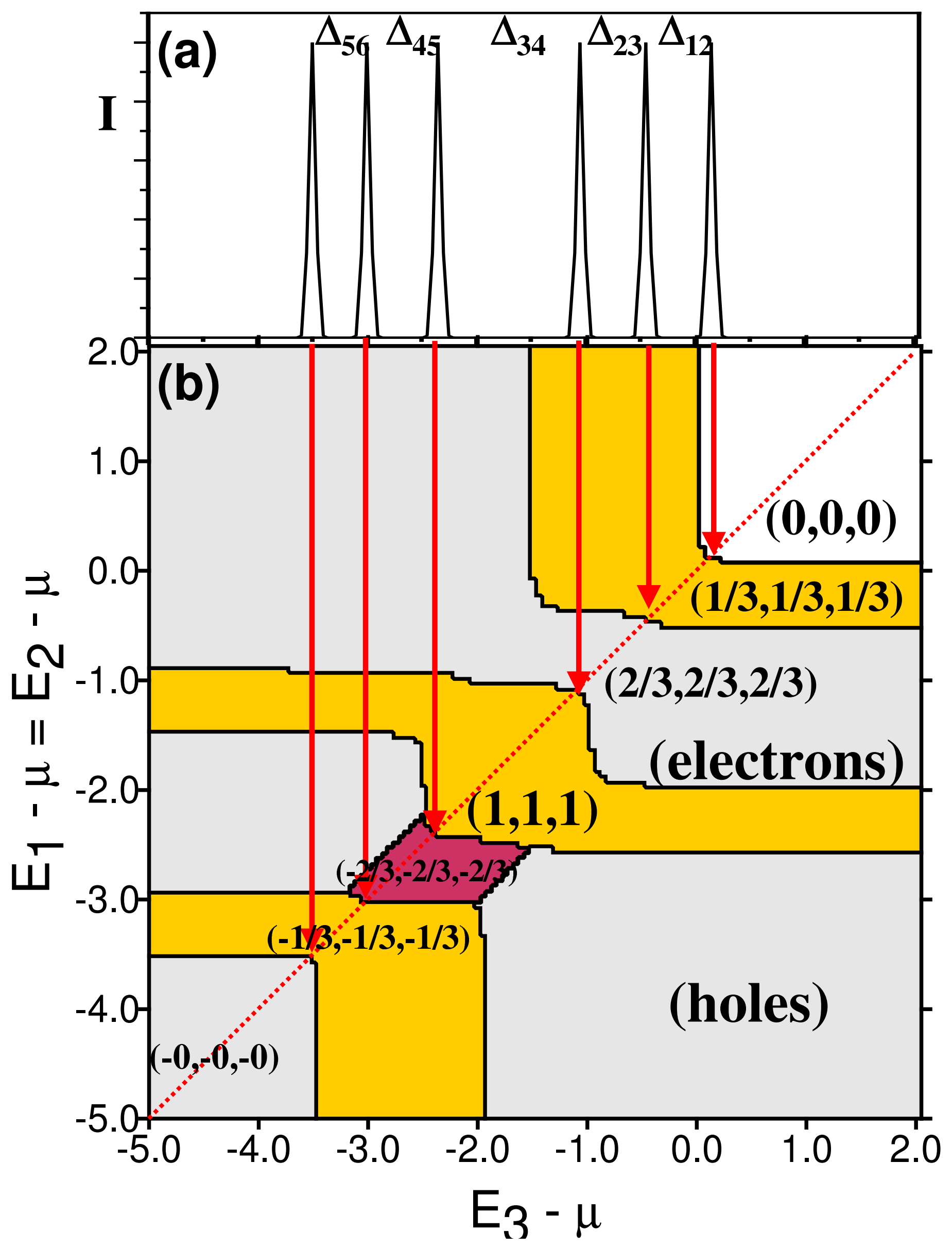




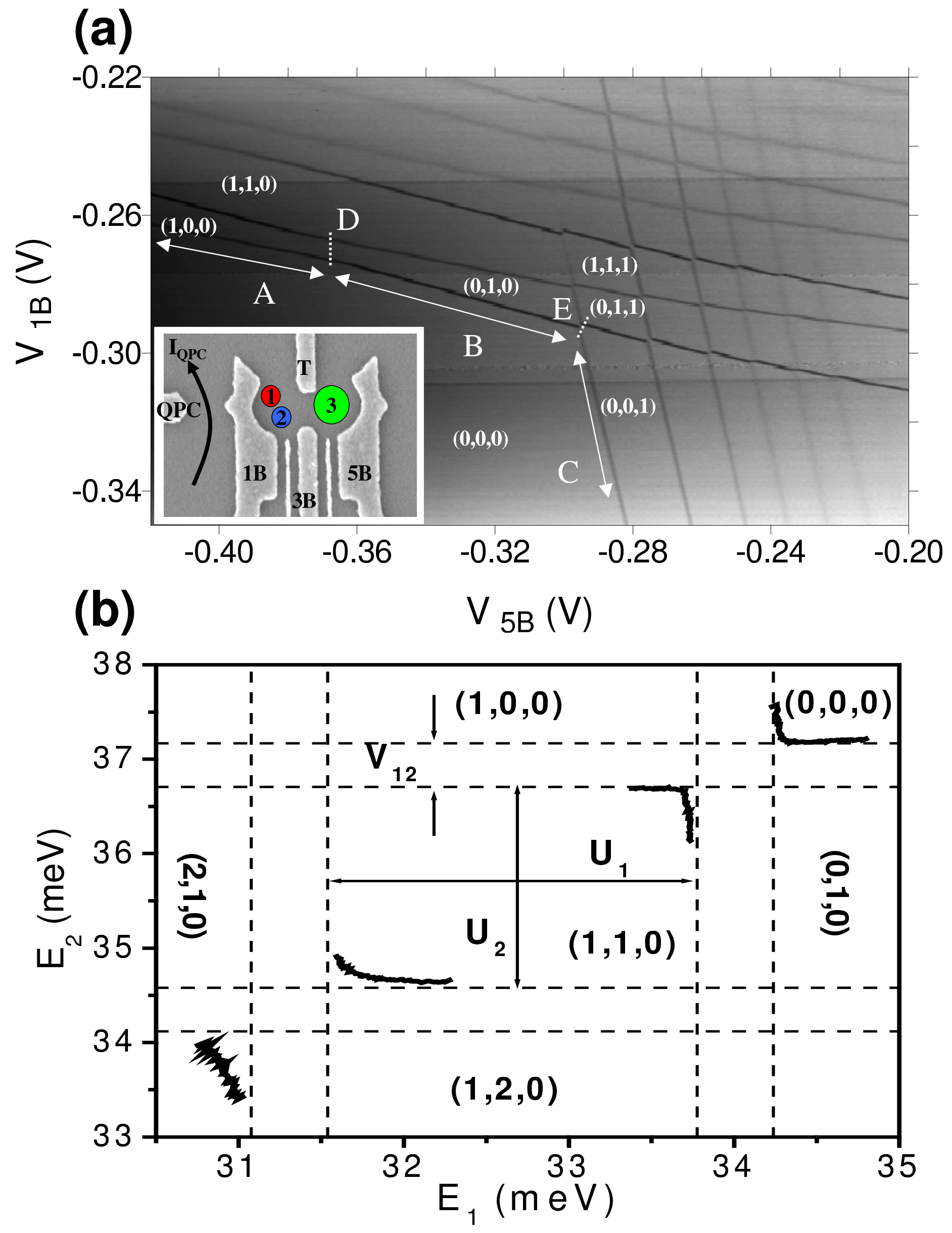




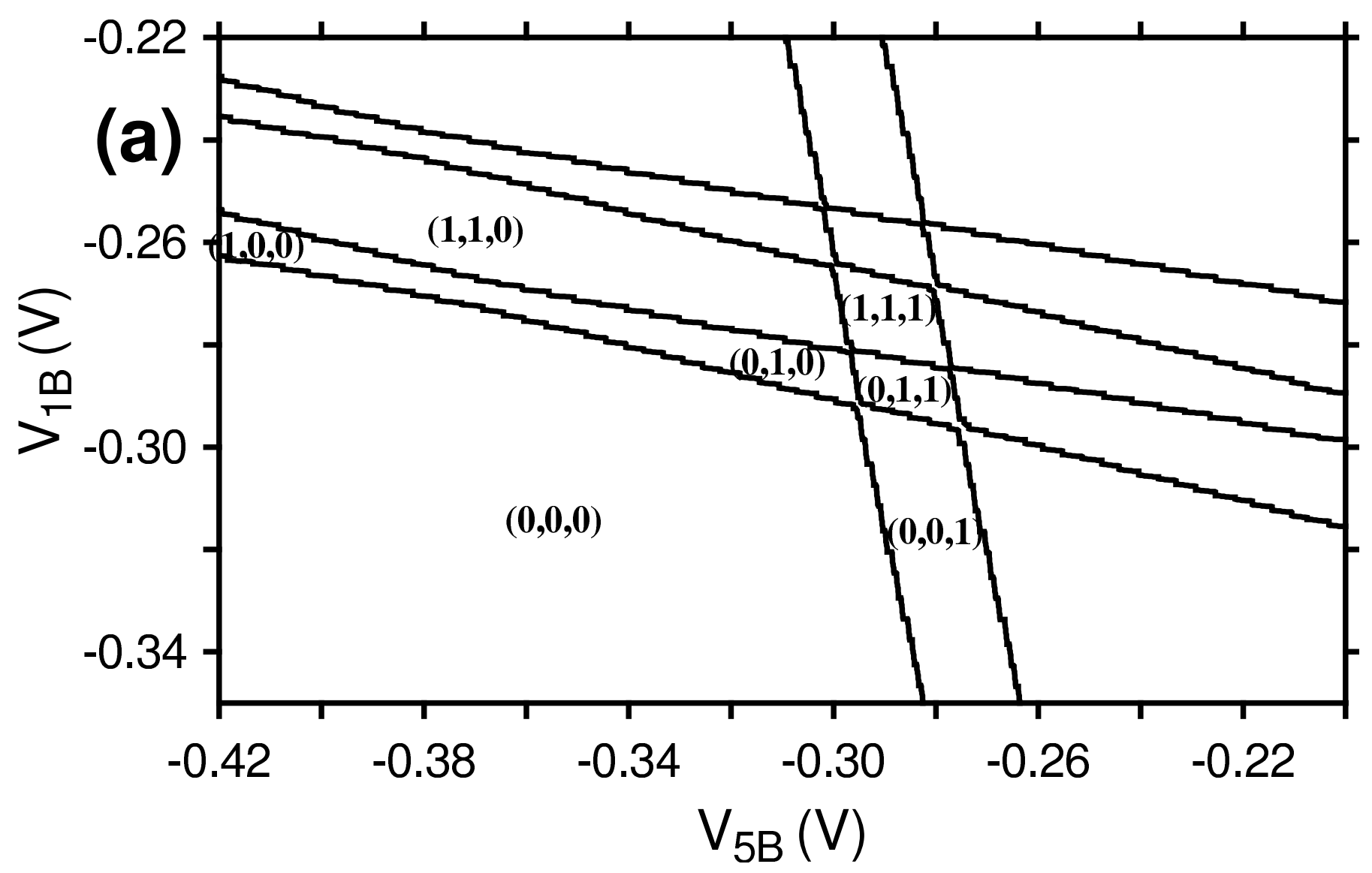

(b)



